\title{
Differential diagnosis of orbital tumors in children
}

\begin{abstract}
Orbital tumors in children are very rare and radiologic image, as magnetic resonance, is extremely important for correct diagnosis. We report six cases of different diagnosis of orbital tumors, comparing their images and clinical aspects. Radiologic images are essential for determine specific diagnosis in most cases of pediatric orbital pathology.
\end{abstract}

Keywords: orbital, tumors, proptosis, hemangioma, rhabdomyosarcoma, neuroblastoma, chloroma, synovial sarcoma, magnetic ressonance imaging, radiology
Volume 3 Issue 4 - 2017

\section{Monica C Rodrigues, Vivian S Tostes, Eliana M Caran, Marcos V Camargo, Frederico Adolfo Silva, Maria Lucia Lee, Carolina AS Santana, Henrique Lederman \\ Department of Radiology, Universidade Federal de Sao Paulo, Brazil}

Correspondence: Henrique Lederman, Department of
Radiology, Universidade Federal de Sao Paulo, Instituto
de Oncologia Pediátrica-GRAACC / UNIFESP, Brazil, Tel
(55) I I 50808586, Email henrique.lederman@gmail.com

\section{Abbreviations: MRI, magnetic resonance imaging \\ Introduction}

Orbital tumors in children are very rare and can initially manifest with ocular proptosis. These lesions have different etiologies, which are divided into benign or malignant, and can be primary from orbital or be a metastatic product. Radiology study of these tumors is extremely essential for distinguish possible diagnosis, once it characterize its localization, extension, relation between adjacent structures, as well as cellularity and vascularity. In this way, radiology studies permits thinking about differential diagnosis easily. We report some clinical cases that have in common orbital commitment and proptosis as first manifestation, and their images correlations in cranial and orbit magnetic resonance. By this, radiological differences between the possible diagnosis will be illustrated.

\section{Case reports}

\section{Case I}

A one-year old boy, presented with a progressively enlarging mass in right eye, with a four months growth. At physical examination, right orbital lesion, with pseudo proptosis, without inflammatory signs or local lymph nodes. Blood count normal, chest X-ray normal, abdominal ultrasound normal. Cranial and orbit MRI shows a right orbital extraconal mass in the upper quadrant invading the eyelid. Biopsy of the lesion showed a embryonal rhabdomyosarcoma Figure $1 \& 2$.

\section{Case 2}

A seven-year old girl, presented with frontal headache, superior left eyelid with ptosis, and blindness, which began fifteen days ago. At physical examination, left eye proptosis, without inflammatory signs or local lymph nodes. Blood count normal, chest X-ray normal, abdominal ultrasound normal. Cranial and orbit MRI reveals a left posteromedial orbital mass with aggressive features extending to the left middle cranial fossa, suggestive of malignant etiology. Incisional biopsy of the lesion revealed a rhabdomyosarcoma (Figure 3).

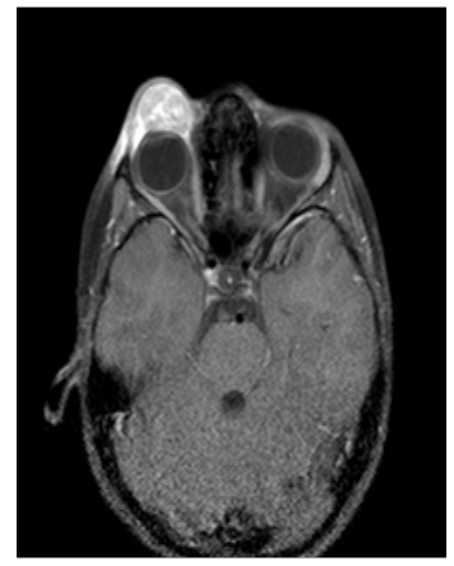

Figure I Axial and sagittal post-contrast TI magnetic resonance imaging (MRI) shows a right well-circumscribed extraconal mass in the upper orbital quadrant with homogeneous enhancement previously invading the eyelid causing marked edema.

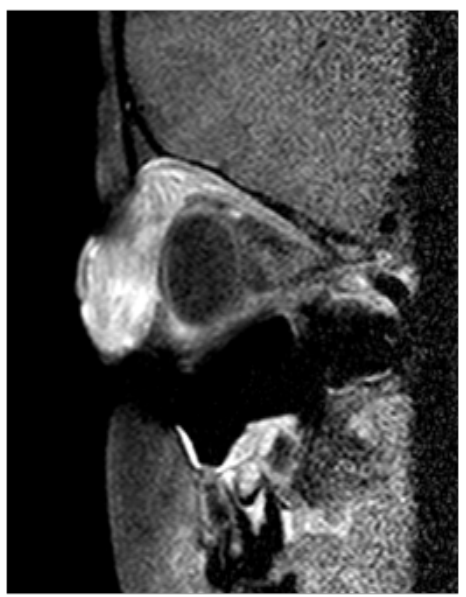

Figure 2 Posteriorly extending through the extraconal space. Note the cortical thinning of superior orbital wall and posterior compression of the globe (embryonal rhabdomyosarcoma). 


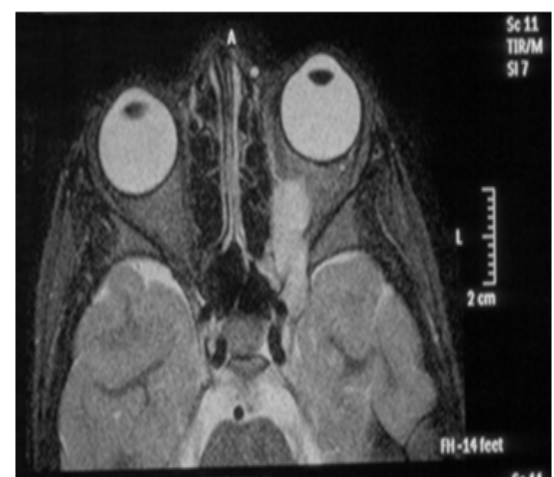

Figure 3 MRIAxialT2 imaging shows a hyperintense left posteromedial orbital mass with extension to the left middle cranial fossa (rhabdomyosarcoma).

\section{Case 3}

A ten-month boy, presented with periorbital edema and proptosis in right eye, two weeks ago. Presented with fever in the last three days. At physical examination, right eye proptosis and inferior eyelid ecchymosis, without inflammatory signs or local lymph nodes. Blood count normal, chest X-ray normal. Abdominal ultrasound showed a left supra-renal heterogeneous mass separated from the kidney and spleen, suggestive of neuroblastoma. Abdominal CT imaging correlates with the ultrasound fidings and cranial and orbit MRI showed a soft-tissue mass along the right greater sphenoid wing and lateral orbital with extension into the orbital compartment causing proptosis and inferomedial displacement of the orbital globe suggestive of metastatic dissemination. Complementary exams were requested: bilateral myelogram, urinary VMA/HVA, MIBG scintigraphy and orbital mass biopsy confirms the diagnosis of neuroblastoma (Figure 4-8)

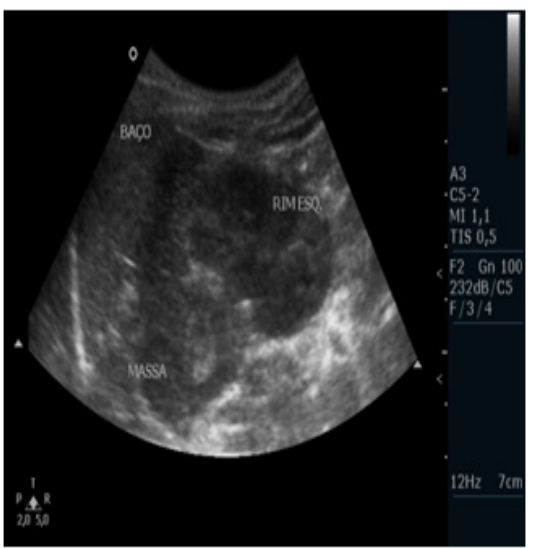

Figure 4 Ultrasound shows a lobulated mass in the left splenorenal space with heterogeneous echogenicity. The lesion is totally separated from the spleen and upper pole of the left kidney.

\section{Case 4}

A nineteen-year old girl, with superior right eyelid edema, which turned into progressive ocular proptosis and visual turbidity, during five months. At physical examination, right eye proptosis, without inflammatory signs. Blood count normal, chest X-ray normal, abdominal ultrasound normal. Cranial and orbit MRI shows a right orbital homogeneous mass involving the intra and extraconal space with extension to the eyelid and temporal scalp with homogeneous contrast enhancement. Incision biopsy of the lesion revealed a granulocytic sarcoma.(primary presentation) (Figure 9-11).

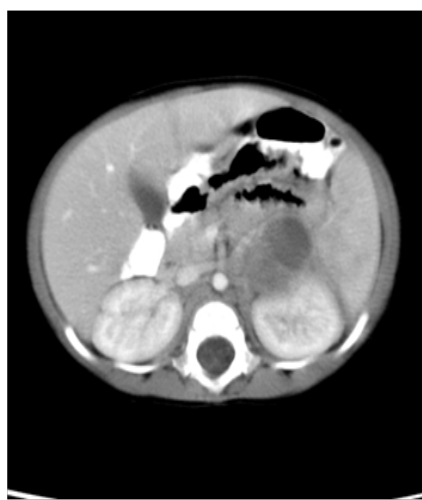

Figure 5 Axial contrast-enhanced CT scan through the upper abdomen reveals a heterogeneous mass anteromedial to the left kidney, with some rounded areas of low attenuation representing cystic necrotic changes.

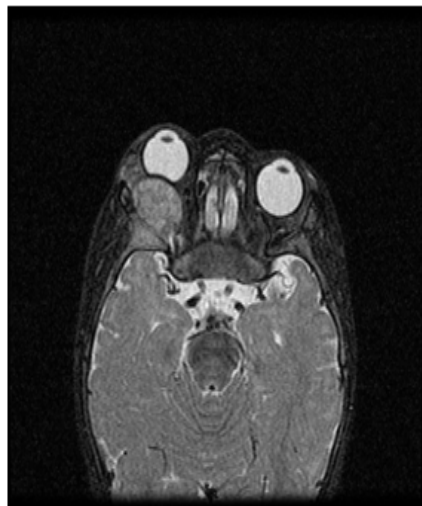

Figure 6 MRI imaging on axial T2 image.

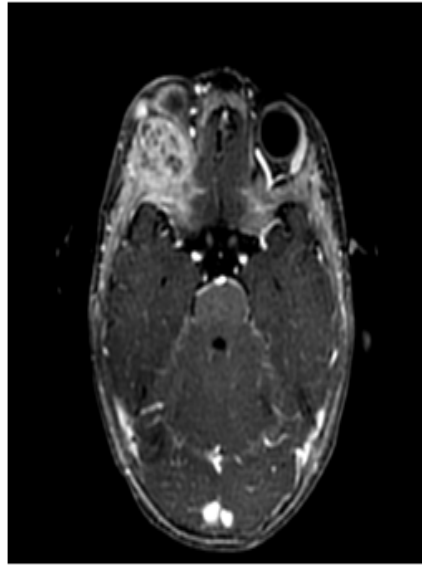

Figure $7 \mathrm{An}$ right iso/hyperintense soft-tissue mass along the greater sphenoid wing and lateral orbital wall with heterogeneous enhancement.

\section{Case 5}

A three-year old girl, presented with periorbital edema for three months, without visual disturbance. At physical examination, right ocular proptosis, without inflammatory signs. Blood count normal, thorax X-ray normal, abdominal ultrasound normal. Cranial and orbit MRI showed a well circumscribed right orbital intraconal lesion with markedly hyperintense on T2 - weighted and enhances brightly on $\mathrm{T} 1$ post contrast suggesting retro-orbital capillary hemangioma. The lesion was immediately behind the eyeball causing flattening of the globe and mild proptosis. Because of its localization and possible bleeding, no biopsy was realized. (Figure 12-14). 


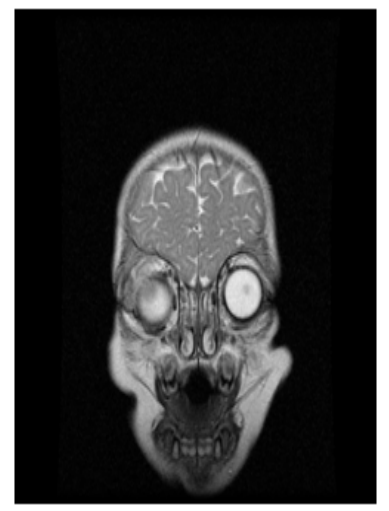

Figure 8 There is extension into the orbital compartment causing proptosis and inferomedial displacement of the orbital globe (neuroblastoma).

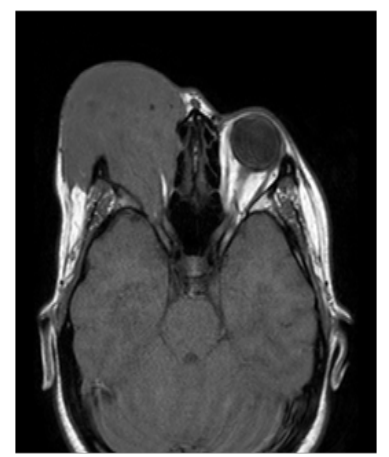

Figure $9 \mathrm{MRI}$ imaging on axial TI.

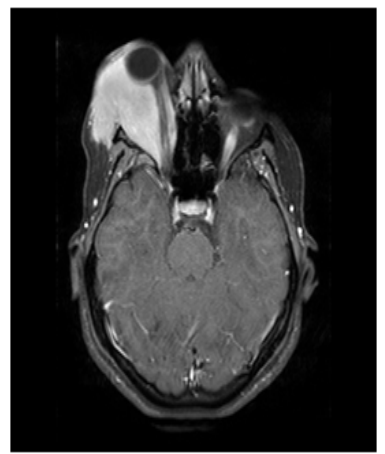

Figure 10 post-contrast.

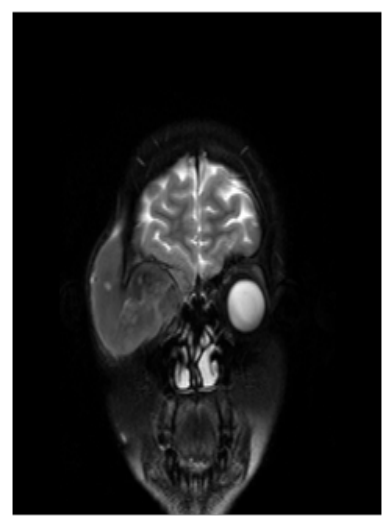

Figure II coronal T2 shows a right orbital mass involving the intra and extraconal space with extension to the eyelid a temporal scalp (granulocytic sarcoma).

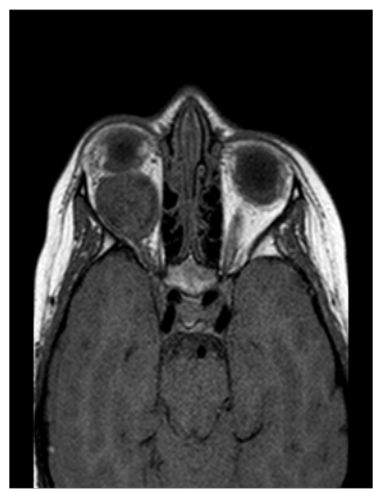

Figure 12 MR images show a well circumscribed right intraconal mass isointense on TI.

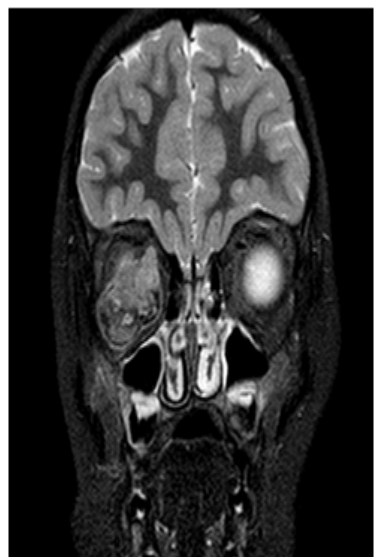

Figure I 3 Markedly hyperintense on T2.

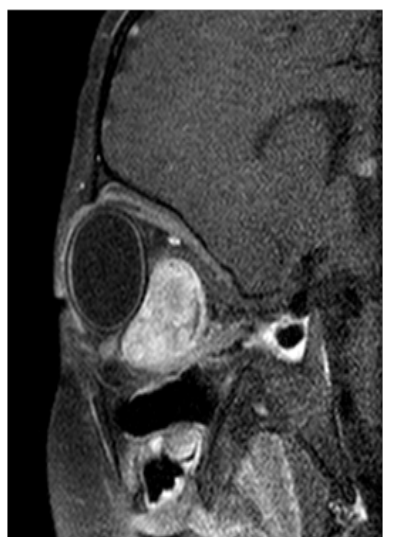

Figure I4 Enhances brightly on TI post contrast. The lesion is immediately behind the eyeball causing flattening of the globe and mild proptosis (capillary hemangioma).

\section{Case 6}

A fourteen-year old girl, with left periorbital mass and ocular proptosis for three months. At physical examination, left ocular proptosis, lacrimation, but without inflammatory signs or local lymph nodes. Blood count normal, chest X-ray normal, abdominal ultrasound normal. Cranial and orbit MRI demonstrates a T1 and T2 iso/hypointense expansive left ethmoidal sinus mass involving the papyrus lamina and compressing de medial orbital structures with displacement of the orbital globe, suggesting malignancy. Incisional 
biopsy of the lesion revealed a synovial sarcoma of ethmoidal sinus (Figures 15).
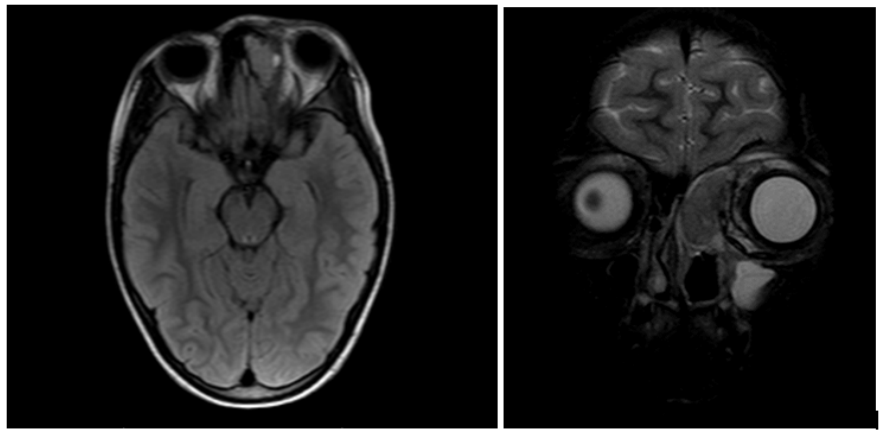

Figure 15 Axial TI and coronal T2 MR imaging shows a iso/hypointense expansive left ethmoidal sinus mass involving the papyrus lamina and compressing de medial orbital structures with displacement of the orbital globe. Note the obstruction of the left infundibuli with secretion retention. (synovial sarcoma of ethmoidal sinus).

\section{Discussion}

Ocular proptosis is the main clinical sign of orbitary pathologies in children. Once clinical condition is related to resonance magnetic images of the lesion, a diagnosis hypothesis may be formulated more precisely. ${ }^{1,2}$

Orbit tumors include a broad spectrum of lesions, which may arise from different orbital structures. They may be classified according to the tissue type of origin, as mesenchymal, neuronal or vascular structures, and may be benign or malignant. From clinical perspective, clinical signs and symptoms are most of them unspecific, as proptosis, diplopia, and optic disco edema, although other symptoms may also be present, as fever, anemia, bone pain. In this way, pediatric oncologists should consider oncology diagnosis for tumors in this localization., ${ }^{2,3}$

MRI has a huge importance in the diagnosis and management of orbitary tumors, because it may characterize lesions in many aspects, as its localization, extension, and relation to other anatomic structures. MRI is the gold standard for evaluate eye and orbit. The best anatomic detail of the orbits is obtained with T1, whereas pathological processes are better characterized on T2 MR images. Furthermore, because the high resolution of the soft tissues, magnetic resonance imaging is the preferred method for delineating orbital processes that many extend intracranial. ${ }^{3-5}$

As benign tumor orbital lesions, capillary hemangioma is the most common orbital tumor in childhood, and is benign lesions. Usually occurs in the anterior region of the orbit. They are not biopsied because of the bleeding risk of the procedure. Differential diagnosis are made with cavernous hemangioma (more common in adults), venous varices and lymphangioma. MRI is suitable for the evaluation and characterization of vascular lesions. They present in the MRI as lesions of heterogeneous intensity in T1 and with high intensity in T2 and flow void is seen. ${ }^{6}$

As malignant lesions, neuroblastoma is the most common extracranial solid tumor in children. The tumor arise from the sympathetic nervous system with intra-abdominal disease representing two-thirds of the cases. Adrenal gland and retroperitoneal (organ of Zuckerland, coeliac axis and paravertebral sympathetic chain) are the main locations. More than fifty percent of cases may have metastatic lesions, mainly localized in bones. Eyelid ecchymosis and proptosis is frequently the first clinical sign of this diagnosis. Children may also present with fever, anemia, and weight loss. The median age is between zero and 4years old, and metastatic lesions are related to a five-year global survival decrease. ${ }^{7,8}$

Rhabdomyosarcoma is the most common mesenchymal neoplasm of orbit in children. Most of the cases occurs sporadically, but few cases are related to genetic syndromes, as Li-Fraumeni. ${ }^{9}$ The median age of presentation is 8-9years old, and the child usually presents with rapidly progressive proptosis, and ocular motility restriction. Thirtyfive percent of these cases are in head and neck region. Most tumors occupy the extraconal space, with approximately 50\% extending intraconal. They may erode bone structures, and extend intracranial, reflecting their aggressive behavior. Children with parameningeal rhabdomyosarcoma may also present with headache, convulsions, visual loss and nystagmus. . $^{910}$

Chloroma, is a soft tissue tumor with leukemic cell infiltrate and may also be called myeloblastoma or granulocytic sarcoma. It represents $3 \%$ of the cases of acute myelogenic leukemia. It is a rare cause of proptosis in children, and most common form of leukemic involvement of the orbit. ${ }^{11,12}$

Synovial sarcoma is a rare malignant soft-tissue neoplasm that accounts for approximately $5 \%$ to $10 \%$ of all soft-tissue tumors. Those that arise from head and neck region is very rare, and sarcoma arising from the orbit is rarer still. Radiology is helpful to characterize the tumor. Magnetic resonance imaging usually reveals a heterogeneous mass with long T1 and long T2 signals. Radiological examinations are useful in the detection of metastatic lesions, which are very important for treatment and prognosis. ${ }^{13}$

\section{Conclusion}

It is important for pediatric oncologists to be aware of the possible different diagnosis for orbital masses. Images study with magnetic resonance play a critical role in the diagnosis and management of patient diagnosis.

\section{Acknowledgements}

None.

\section{Conflict of interest}

Author declares that there is no conflict of interest.

\section{References}

1. Ajay A Rao, John H Naheedy, James YY Chen, et al. A Clinical Update and Radiologic Review of Pediatric Orbital and Ocular Tumors. Journal of Oncology. 2013;2013:1-22.

2. Ro SR, Asbach P, Siebert E, et al. Characterization of orbital masses by multiparametric MRI. Eur J Radiol. 2016;85(2):324-336.

3. Vachha BA, Robson CD. Imaging of Pediatric Orbital Diseases Neuroimaging Clin N Am. 2015;25(3):477-501.

4. Aviv RI, Casselman J. Orbital imaging: Part 1. Normal anatomy. Clin Radiol. 2005;60(3):279-287.

5. Purohit BS, Vargas MI, Ailianou A, et al. Orbital tumours and tumourlike lesions: exploring the armamentarium of multiparametric imaging. Insights imaging. 2016;7(1):43-68. 
6. Calandriello L, Grimaldi G, Petrone G, et al. Cavernous venous malformation (cavernous hemangioma) of the orbit: Current concepts and a review of the literature. Surv Ophthalmol. 2017;62(4):393-403.

7. Kurian Joshua, Neil Chen N, Roman Shinder. Orbital Metastatic Neuroblastoma. Ophthalmology. 2016;123(8):164.

8. Kembhavi SA, Shah S, Rangarajan V, et al. Imaging in neuroblastoma: An update. Indian J Radiol imaging. 2015;25(2):129-136.

9. Radzikowska J, Kukwa W, Kukwa A, et al. Rhabdomyosarcoma of the head and neck in children. Contemp Oncol (Pozn). 2015;19(2):98-107.

10. Chung EM, Smirniotopoulos JG, Specht CS, et al. From the archives of the AFIP: Pediatric orbit tumors and tumorlike lesions: Nonosseous lesions of the extraocular orbit. Radiographics. 2007;27(6):1777-1799.
11. Singh A, Kumar P, Chandrashekhara SH, et al. Unravelling chloroma: review of imaging findings. The British Journal of Radiology. 2017:1075(90).

12. Balyen L, Deniz Balyen LS, et al. Case of Orbital Granulocytic Sarcoma (Chloroma) Well Responded to the Treatment. Adv Ophthalmol Vis Syst. 2016;5(1):00139.

13. Xu P, Chen J. Primary Synovial Sarcoma of the Orbit. Ophthalmol Eye Dis. 2017;9:1-5. 